mortality), morbidity, total disease burden and selected health risks and determinants for 10-24-year-old Malaysians, by three age groups (10-14, 15-19 and 20-24 years) and sex, from 1990 to 2017.

Results While the total disease burden decreased, most of this decrease was due to mortality, with morbidity mostly unchanged for all age groups and sexes between 1990 and 2017. Most of the reductions in disability-adjusted life years (DALYs) appear driven by a reduction of mortality from vaccine preventable diseases and maternal causes, across all age groups. Communicable diseases remain most prevalent in the 10-14-year-olds and some causes (e.g. diarrheal diseases, dietary iron deficiency) have increased by 2017. Morbidity from non-communicable diseases (NCDs) continued to be high in 2017. By 2017, mental disorders (e.g. anxiety, depression, conduct disorders) and chronic physical disorders (e.g. migraine, low back pain) were important contributors of morbidity for all adolescents. Motor-vehicle accidents were the principal cause of death in 15-19 and 20-24-year-olds of both sexes, while drowning and lower respiratory infections were the leading causes of death in 10-14-year-old males and females, respectively. The prevalence of male tobacco smoking had changed little across all groups, highest for males aged 20-24 years at $49.4 \%$ in 1990 and $44.4 \%$ in 2017. In contrast, rates of overweight and obesity had tripled in females and quintupled in males. Rates of youth not in education, employment and training rates had decreased, while adolescent fertility rate has halved from 1990.

Conclusion These data highlight that adolescent specific interventions are inadequate and health actions are required to address the disease burden from NCDs, injuries, some communicable diseases and obesity, which in addition to the health sector, will require multisector actions.

\section{P24 UPTAKE OF THE MENACWY VACCINE AND VACCINATION VIEWS AMONG FIRST-YEAR STUDENTS AT A LONDON UNIVERSITY}

${ }^{1} \mathrm{SE}$ Jones ${ }^{*},{ }^{2} \mathrm{H}$ Bedford, ${ }^{2} \mathrm{M}$ Cortina Borja. ${ }^{1} \mathrm{UCL}$ Great Ormond Street Institute of Child Health, London, UK; ${ }^{2}$ Population, Policy and Practice Programme, UCL Great Ormond Street Institute of Child Health, London, UK

\subsection{6/bmjpo-2019-RCPCH-SAHM.29}

Background New university students are at particular risk of invasive meningococcal disease (IMD). Group W, a particularly aggressive strain, is increasing in prevalence and the high casefatality rate is concerning. This age group has been offered the MenACWY vaccine since 2015. National uptake has been low, leaving students vulnerable to infection.

Aims To investigate MenACWY uptake, knowledge of and attitudes towards vaccination among first-year students, with the aim of informing university vaccination policy and practice.

Methods A mixed methods approach was used, involving a questionnaire (response rate $=4.4 \%, n=144$ ) and follow-up interviews $(n=13)$. Eligibility criteria were first year students, undergraduates and over the age of 18 . Statistical tests, including multiple logistic regression, were carried out and interviews were analysed thematically.

Results MenACWY uptake was 84\%, with more socioeconomically disadvantaged students less likely to be vaccinated
$(\mathrm{aOR}=0.117, p=0.006)$. Most students thought vaccines were safe (95.1\%) and important (97.2\%). Students with above average knowledge were more likely to be vaccinated $(\mathrm{OR}=3.057, p=0.019)$. Students unaware that meningitis can be fatal were less likely to be vaccinated $(\mathrm{aOR}=0.173$, $p=0.035)$. Vaccination views were positive and knowledge level was moderate to high. Reasons for vaccination include influence of authority figures and peers, to avoid disease and due to an inherent trust of vaccines. Reasons for non-vaccination included temporary illness, laziness, forgetfulness and difficulty with GP access. Opinions regarding the university's vaccination campaign were positive, and in particular there was praise for the university's awareness campaign. Issues raised by this study include difficulty in accessing GP services and the belief that the vaccine prevents any cause of meningitis.

Conclusion High vaccine uptake is essential to protect students. Uptake was higher than at other universities in previous studies. These results highlight several areas requiring further study, including the association between uptake and socioeconomic group and understanding of post-vaccination risk of meningitis. This research has implications for vaccination policy at UK universities.

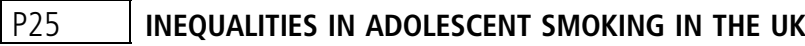 MILLENNIUM COHORT STUDY: ESTIMATING THE RELATIVE CONTRIBUTIONS OF VERBAL ABILITY AND SELF-REGULATION}

H Creese*, R Viner, S Hope, A Pearce, A Ross. Population, Policy and Practice, University College London, London, UK

\subsection{6/bmjpo-2019-RCPCH-SAHM.30}

Smoking is a leading cause of morbidity and preventable death in the UK. Adolescence is a time when smoking behaviours are often initiated, therefore it is important to understand potential risks and protective factors. Higher cognitive development in adolescence is related to a lower likelihood of smoking. Cognitive development is characterised by the growth of abilities and skills in multiple domains, including verbal ability. Similarly, higher 'non-cognitive' ability has been associated with lower levels of smoking. One element of 'non-cognitive' ability is self-regulation which refers to an individual's control of thoughts, emotions and behaviour in order to achieve a goal. Socioeconomic disadvantage is associated with higher rates of initiation and progression to daily smoking. In addition, verbal ability and self-regulation are generally lower in socioeconomically disadvantaged children. We investigated whether the relationship between early socioeconomic circumstances and adolescent smoking was partially driven by cognitive development and/or self-regulation. Using an Oaxaca-Blinder decomposition model, we estimated the relative contributions of verbal ability and/or self-regulation at 11 years to the association between early life socioeconomic disadvantage captured by household poverty status at 9 months and smoking in adolescence (at 14 years) in the UK Millennium Cohort Study $(\mathrm{N}=6,737)$. Verbal ability was assessed using a validated, age-appropriate test, the British Ability Scales Second Edition (BAS 2). Five parent-rated items from the Strengths and Difficulties Questionnaire were 
used to capture two components of self-regulation - task attentiveness and emotional regulation. Children who had lived in poverty at 9 months were almost twice as likely to smoke at age 14. Twelve per cent of the difference in smoking between poverty groups was attributable to self-regulation, with none explained by verbal ability. The remaining difference was largely due to socio-demographic factors. Interventions to improve self-regulation targeted at children from families experiencing socioeconomic disadvantage at the end of primary school may hold promise to reduce inequalities in smoking. However, the detrimental impact of early life poverty on smoking in adolescence is chiefly driven by socio-demographic factors rather than later, individual characteristics such as self-regulation.

\section{P26 EXPLORING THE EXPERIENCE OF ADOLESCENTS WITH DOWN SYNDROME AND THEIR PARENTS}

F McGrane*, EF Roche. 'Discipline of Paediatrics, Trinity College, Dublin, Dublin, Ireland; ${ }^{2}$ Department of Paediatrics, Children's Health Ireland, Tallaght, Dublin, Ireland

\subsection{6/bmjpo-2019-RCPCH-SAHM.31}

Aims Research regarding adolescents' with Down syndrome (DS) is limited. The majority of previous studies have been undertaken in either the UK or USA but none in Ireland. This study sought to address this gap by exploring the experience of growing up in Ireland for adolescents with Down syndrome and their aspirations for the future.

Methods The study was conducted using two questionnaires designed for administration to adolescents with Down syndrome and self-completion by parents. Areas explored in detail included health, service provision, education, living circumstances and future aspirations.

Results Forty seven adolescents with DS participated, aged 12-18 years (23 female) and 49 parents. The frequency of health comorbidities were assessed, $87 \%$ felt they had good health with eyes, hearing and heart. Further detail was obtained in key specific health areas. Daily medication was taken by $44 \%$. A large proportion of parents $(45 \%)$ and adolescents (98\%) were unaware of the DS medical management guidelines. Parents were asked if their adolescent received satisfactory services across the domains. Parental reported satisfaction with care received in vision (92\%), hearing (90\%), medical care (86\%) dietetics $(55 \%)$ and speech and language therapy (39\%). Dissatisfaction related to access rather than care received. There was limited use of respite services (30\%). There was a negative perception of respite amongst some parents (17\%) although those who availed of respite had largely positive experiences (92\%). Transition of care from paediatric to adult services is an area of concern for the majority of parents $(63 \%(n=31))$ but not for adolescents. Most adolescents $(79 \%)$ were unaware of transition to adult services and were unconcerned by it. Parents felt transition could be improved by additional information (61\%), increased preparation $(51 \%)$ and planning and discussion with adolescents $(63 \%)$.

Conclusion The study showed overall satisfaction in relation to health and service provision but highlighted concerns regarding access to critical time sensitive services. Of particular concern there was poor awareness of the Medical management guidelines.

\section{P27 \\ WHEN CHRONIC FATIGUE SYNDROME LEADS TO MUTISM}

${ }^{1,2} \mathrm{~S}$ Moeda*, 1,3 L Gamper, ' A Gregorowski, 'T Segal. 'Children and Young People's Specialist Adolescent Services, University College Hospital, London, UK; ${ }^{2}$ Department of Paediatrics, Hospital Beatriz Ângelo, Loures, Portugal; ${ }^{3}$ Department of Paediatrics, University Children's Hospital, Zurich, Switzerland

\subsection{6/bmjpo-2019-RCPCH-SAHM.32}

Aims Chronic Fatigue Syndrome (CFS) impacts negatively the quality of life, school attendance and social and family functioning. Cognitive dysfunction represents one of the most common and disabling symptoms. We report a severe case of CFS with extreme cognition loss.

Methods Case-report.

Results A previously healthy and athletic male adolescent developed weakness and fatigue following a viral illness at 10 years old. Mobility progressively deteriorated over two years, requiring wheelchair and admission. Investigations, including brain and spinal MRI, were unremarkable and CFS diagnosed. $\mathrm{He}$ started intensive physiotherapy but, while his physical activity improved, an abrupt extreme cognitive loss took place. He reported 'brain fog' and formal testing showed incredibly slow cognitive and sensory processing (e.g. score of 4/10 in processing incoming language). After little improvement, he restarted deteriorating and stopped attending school.

Aged 16, he was referred to our specialist CFS team, lying mainly in bed and unable to feed himself. He had diminished ability to speak and slow comprehensive processing. $\mathrm{He}$ struggled with significant anxiety, low mood, poor sleep and socially isolation. Further investigation, with EEG and neurological reassessment, was normal. He initiated a regular multidisciplinary rehabilitation program, including patient and family support, pacing, graded exercise, sleep hygiene and pain relief. He indicated that he chose to use his energy on physical improvement at the expense of communication, hence, while his mobility slowly improved over one year, his cognitive ability notably deteriorated. He became gradually more non-verbal and uncommunicative, initially using signboards, but then refusing. Once his physical activity had markedly improved from being bedbound to walking, he restarted communicating by smiling and using thumbs up/down, slow speech with lags between words, then rapidly increasing to full sentences. After two years of therapy his improvement has been remarkable. Currently he walks, sleeps regularly, speaks fluently and engages with family and friends. He has home- tuition and has future goals.

Conclusion Establishing the accurate diagnosis and early symptom management are crucial for rehabilitation in CFS. Cognitive impairment may be significantly debilitating. Physical and cognitive recovery occurs at different stages. A multi-disciplinary patient-centred approach, working with families and local services, can be very effective.

\section{P28 IMPROVING TRANSITION FOR PATIENTS WITH DIABETES: A SERVICE IMPROVEMENT PROJECT}

F Fuller*, R Gadsby. Warwick Medical School, Warwick University, Warwick UK

\subsection{6/bmjpo-2019-RCPCH-SAHM.33}

Aims To examine what adolescents with Type 1 diabetes want from a transition service and to evaluate if the implementation of a Specialist Transition Nurse improves patient engagement 